\title{
Velocity-matched electro-optic modulator
}

\section{William B. Bridges, Finbar T. Sheehy, James H. Schaffner}

William B. Bridges, Finbar T. Sheehy, James H. Schaffner, "Velocity-matched electro-optic modulator," Proc. SPIE 1371, High-Frequency Analog Fiber Optic Systems, (1 February 1991); doi: 10.1117/12.24884 
Velocity-Matched Electro-optic Modulator*

\author{
William B. Bridges and Finbar T. Sheehy \\ California Institute of Technology, \\ Pasadena, CA 91125 \\ and \\ James H. Schaffner \\ Hughes Research Laboratories, \\ Malibu, CA 90265
}

\begin{abstract}
$\underline{\text { ABSTRACT }}$
Partitioning the transmission line on a $\mathrm{LiNbO}_{3}$ traveling wave modulator into short sections and feeding each section from an antenna integrated on the modulator surface can overcome the frequency limitation from material dispersion. We have demonstrated a phase modulator at $5-13 \mathrm{GHz}$ with a peak sensitivity better than 100 degrees/(Watt) $)^{1 / 2}$ using an unoptimized microwave feed system. The technique should be scalable to high millimeter-wave frequencies.
\end{abstract}

\title{
1. INTRODUCTION
}

Electro-optic modulators using $\mathrm{LiNbO}_{3}$ have been demonstrated with modulation frequencies over $17 \mathrm{GHz}$ and with modulation voltages less than $1 \mathrm{~V}^{1,2}$. Unfortunately there is a trade-off between high sensitivity and high-frequency operation. While $\mathrm{LiNbO}_{3}$ has a high electro-optic coefficient, it is also dispersive, having a much higher refractive index at microwave frequencies than for optical signals. The consequent mismatch of optical and microwave phase velocities limits the useful interaction length to about one-quarter free-space wavelength at the microwave frequency. If the modulator is long, for high sensitivity, then the maximum modulation frequency is low: if the modulation frequency is high then the modulator must be kept short, limiting sensitivity. We have demonstrated a new technique which overcomes this limitation and, as an added benefit, uses a waveguide to introduce the modulation signal. This latter feature may prove as important as the former for modulators operating in the millimeter wave range.

* This work was supported by a grant from the Caltech President's Fund and a grant from Rome Air Development Center. 
There have been previous approaches to overcoming the material dispersion limitation. Alferness et al. ${ }^{3}$ have demonstrated a technique in which the modulation electrodes are divided into sections, each of which introduces a phase error of $180^{\circ}$ between the optical and modulating signals. The sections are then connected together so as to correct the phase by $180^{\circ}$. In this way the average phase velocities of the two signals are kept equal. Some loss of sensitivity does occur because the electrode length which is required to introduce the $180^{\circ}$ phase error is quite long.

Schaffner ${ }^{4}$ has divided the electrodes into shorter sections to obtain greater sensitivity. The sections are connected by stub transmission lines that provide a phase delay of $360^{\circ}$ minus the phase error introduced by each short electrode section. As in reference 3 the average phase velocity of the modulating signal is kept equal to that of the optical signal, but the shorter sections improve the sensitivity.

Our technique also divides the modulator into sections which limit the phase error per section. Now the individual sections are connected to surface antennas, as shown in Figure 1. The antennas are illuminated by a plane wave (the modulating signal) at an angle which provides the required phaseshift between the antennas. Since the $\mathrm{LiNbO}_{3}$ has a high value of $\epsilon_{r}$ the antennas are much more sensitive to radiation incident from inside the substrate than from outside ${ }^{5}$. The required angle of incidence for the plane-wave illumination is $\theta=\sin ^{-1}\left(\mathrm{n}_{o} / \mathrm{n}_{m}\right)$ where $\mathrm{n}_{o}$ is the optical refractive index and $\mathrm{n}_{m}$ is the microwave refractive index of $\mathrm{LiNbO}_{3}$.

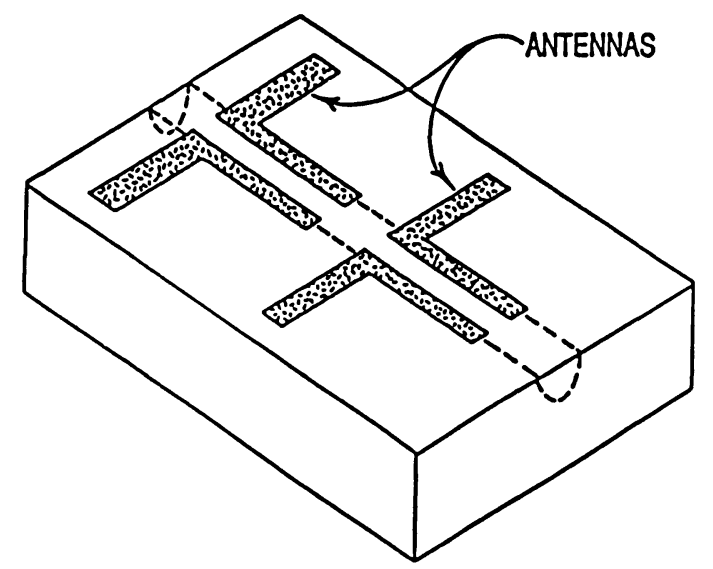

Figure 1. Schematic drawing of two dipole antennas and connected transmission line sections on the surface of a waveguide $\mathrm{E}-\mathrm{O}$ modulator.

Since no physical connections are made to the antennas and the short modulating elements need not be terminated by matched loads (although they could be), no parasitic circuit elements (such as bond wires or connectors) are introduced which might limit the possibility of scaling to higher frequencies. The limits would be imposed by the size of the optical waveguide and by the absorbtion 
spectrum of the $\mathrm{LiNbO}_{3}$ itself. A modulator at $500 \mathrm{GHz}$ should be quite practical. In addition the problem of attenuation along a long modulator does not exist, since the elements are driven "from the side", in parallel rather than in series. In exchange for these advantages, we must develop efficient means for illuminating the modulator.

\section{EXPERIMENTAL PHASE MODULATOR}

Figure 2 shows the mask for an X-band prototype modulator. There are five antenna/modulator sections. The overall length is $25 \mathrm{~mm}$. The antennas are configured as "two half-waves in phase", and

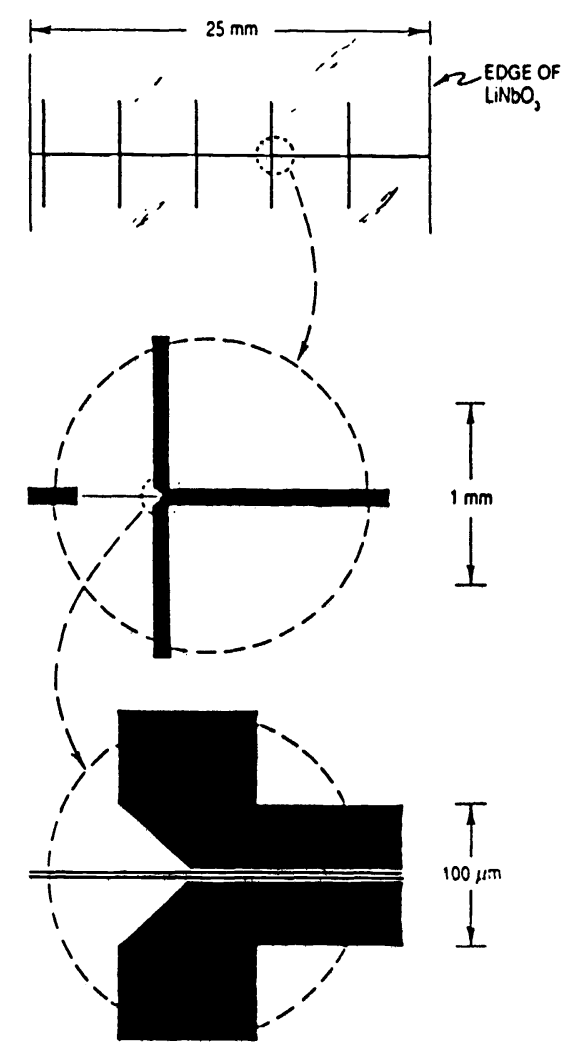
the modulator sections are approximately a half-wave long at $10 \mathrm{GHz}$, based on an assumed effective microwave surface refractive index of 4.3 (i.e. the dielectric constant is taken as the mean of the dielectric constants of air and $\mathrm{LiNbO}_{3}$ ). This electrode pattern was positioned over a straight section of optical waveguide to produce a phase modulator rather than a Mach-Zehnder amplitude modulator, but the same mask would also work if applied to one arm of a Mach-Zehnder. The $6 \mu \mathrm{m}$ optical waveguide was produced by indiffusion of titanium.

Figure 2. Photolithographic mask for 5 antennaplus-transmission lines covering a $25 \mathrm{~mm}$ long optical waveguide. Magnified details are shown in the lower two drawings.

The microwave feed used to drive the modulator is shown in Figure 3. The $\mathrm{LiNbO}_{3}$ substrate is attached to a wedge of Stycast ${ }^{\mathrm{R}}$ dielectric with $\epsilon_{r}=30$, and the signal is matched into this material by two matching layers of intermediate dielectric constant. The wedge angle is $23^{\circ}$ to match the 
microwave and optical phase velocities: a wedge is necessary because the critical angle is only $9^{\circ}$, making it impossible to couple into the substrate at any larger angle without using a wedge. The optical beam at $633 \mathrm{~nm}$ is coupled into and out of the optical waveguide using microscope objectives. Optical damage occurs in the guide at $633 \mathrm{~nm}$, and optical power was limited because of this.

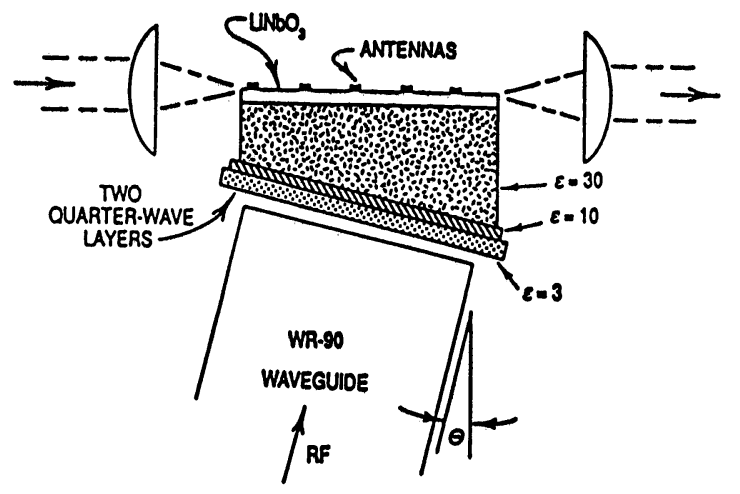

Figure 3. Schematic side view of an experimental modulator showing $\mathrm{LiNbO}_{3}$ wafer with antennas, input and output lenses, wedged block of high dielectric constant material, matching layers, and microwave waveguide.
As was mentioned above, the pattern of an antenna on a dielectric substrate is directed more into the substrate than into the air. For $\epsilon_{r}=30$ there is virtually no coupling on the air side. The pattern in the dielectric is also distorted by the presence of the interface. There is a peak response in the direction of the critical angle (here $9^{\circ}$ ). We used the theory of Enghetta et al. ${ }^{3}$ to evaluate the pattern at the desired angle of $23^{\circ}$, and find that it is only about $0.5 \mathrm{~dB}$ down from the peak (Fig. 4).

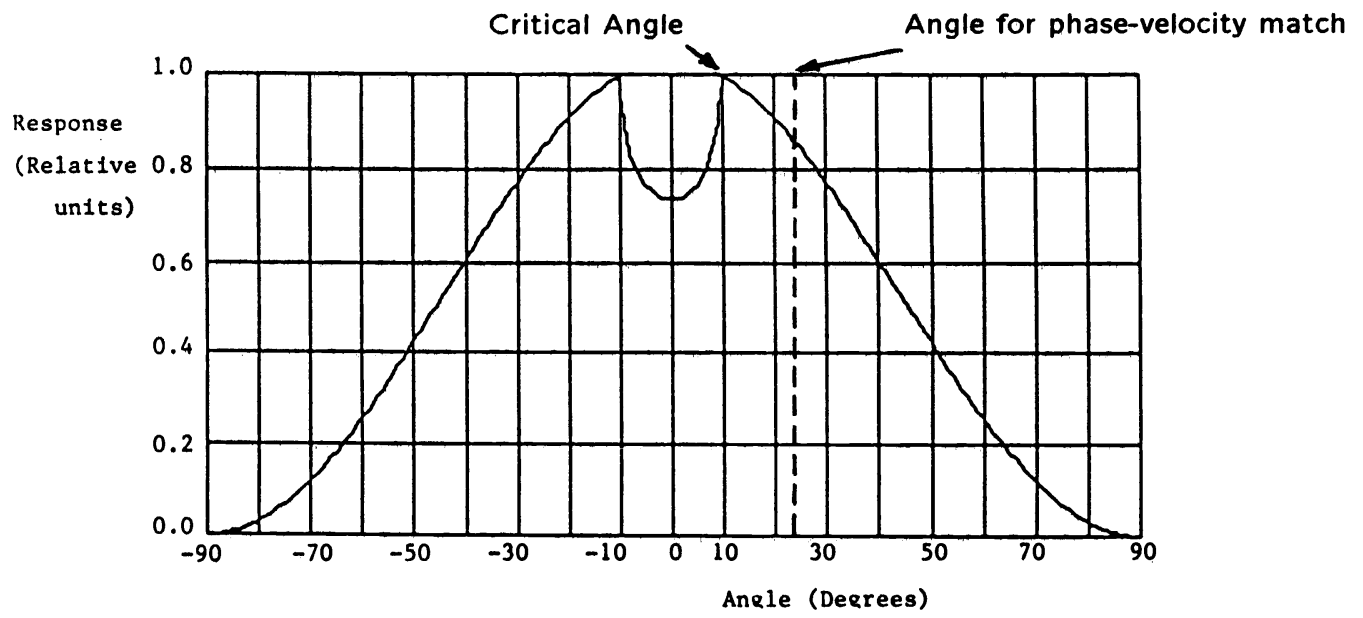

Figure 4. Antenna pattern of Dipole on $\mathrm{LiNbO}_{3}$. (The cusp-like behavior at the critical angle is a real feature.)

The phase modulated signal cannot be detected using an amplitude detector. We used a scanning Fabry-Perot interferometer to show the existence of modulation sidebands and measure their amplitude. The phase deviation introduced by the modulator can be deduced from the amplitude of 
the sidebands relative to the carrier (for small phase deviation) from the formula ${ }^{6}$

$$
\Delta \phi=2 \sqrt{\frac{\mathrm{P}_{\text {sideband }}}{\mathrm{P}_{\text {carrier }}}}
$$

It is more usual to build an amplitude modulator and detect the signal directly, but the above method is simple and has the advantage that it works at much higher modulation frequencies, where a-m detectors are scarce. It has one notable disadvantage, however. The frequency spectrum is aliased by the scanning Fabry-Perot at frequency intervals equal to the free spectral range, so that sidebands which are an integral number of free spectral ranges off carrier are lost behind an aliased version of the carrier.

Figure 5 shows the modulator response as a function of frequency. The response is here defined as phase deviation normalized to the square root of the drive power, in units of

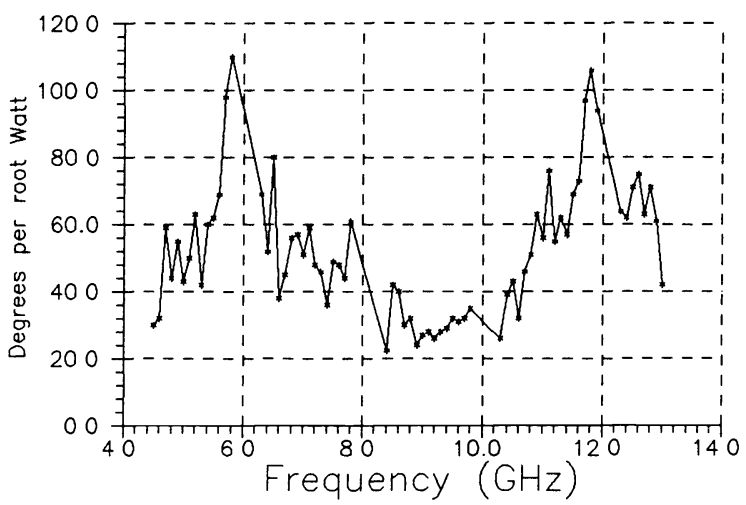

Figure 5. Frequency response of the experimental modulator. "degrees per root Watt". This measure is linearly related to "degrees/radians per Volt", which is the common measurement when direct connections are made and voltage can be measured directly. There are no data points at $6,8,10$ and $12 \mathrm{GHz}$ due to the aliasing of the carrier at $2 \mathrm{GHz}$ intervals making the sidebands invisible on the oscilloscope display. The graph shows straight-line interpolation between the data points.

\section{DISCUSSION OF $\underline{\text { RESULTS }}$}

The structure of the curve arises from many sources: antenna and electrode resonance; reflections within the wedge; nonideal input matching layers; etc. However, we have found that the frequency response is reasonably well accounted for by a model of the antenna/electrode impedance variations and the frequency response of the individual modulators. In this model the electrodes are treated as lossless transmission lines, open-circuit at the end; the antenna is treated as a lossy transmission line (the loss represents the radiation resistance); the individual modulators have frequency response 
basically determined by the well-known sinc-function response, except that there are two sinc terms, one for the forward-propagating wave at the modulation frequency and one for the wave reflected back from the open circuit at the end of the modulator electrodes. The model therefore uses the following equations:

$$
\text { Antenna Impedance: } \quad \mathrm{Z}_{\text {ant }}=\mathrm{R}+\mathrm{j} \mathrm{X}=\frac{95}{\tanh (0.157 \theta(\mathrm{f})+\mathrm{j} \theta(\mathrm{f}))}
$$

where $\theta(f)$ is the electrical half-length of the antenna at

$$
\text { frequency } \mathrm{f} \text {. }
$$

Electrode Voltage: $\quad \mathrm{V}_{f w d}(\mathrm{f})=\frac{\mathrm{Zo} \sqrt{\mathrm{R} \mathrm{P}_{i n c} \mathrm{~A}}}{\sqrt{\mathrm{R}^{2} \sin ^{2}(\theta)+[\mathrm{X} \sin (\theta)-\mathrm{Zoc} \cos (\theta)]^{2}}}$

where Zo is the electrode/transmission line impedance,

$\mathrm{P}_{\text {inc }}$ is the incident power density,

and $\quad A$ is the antenna effective area.

The antenna effective area is given by

$$
\mathrm{A}=\frac{\lambda^{2} \mathrm{G}}{4 \pi}
$$

where $\lambda$ is the wavelength (in the dielectric),

and $\quad G$ is the gain at the angle of incidence, relative to an isotropic radiator. We believe that the gain varies relatively slowly as a function of wavelength, by a factor of 2 or so for the range in question.

Modulator Frequency Response:

$$
\delta(\mathrm{f})=\frac{2 \pi \mathrm{fr}_{33} \mathrm{n}_{o}{ }^{3} \mathrm{E}_{z}}{2 \mathrm{c}} \mathrm{L}\left[\operatorname{sinc}\left(\frac{2 \pi \mathrm{f}\left(\mathrm{n}_{o}-\mathrm{n}_{m}\right) \mathrm{L}}{2 \mathrm{c}}\right)+\operatorname{sinc}\left(\frac{2 \pi \mathrm{f}\left(\mathrm{n}_{o}+\mathrm{n}_{m}\right) \mathrm{L}}{2 \mathrm{c}}\right)\right]
$$

where $\mathrm{n}_{o}$ is the optical refractive index,

$\mathrm{n}_{m}$ is the effective microwave refractive index,

$c$ is the speed of light,

$r_{33}$ is the electro-optic coefficient for the material, 
$\mathrm{E}_{z}$ is the electric field strength between the electrodes, and $L$ is the electrode length.

Then the frequency response of the modulator is

$$
\mathrm{M}(\mathrm{f})=\delta(\mathrm{f}) \times \mathrm{V}_{f w d}(\mathrm{f})
$$

The frequency-response which is predicted by the simple model of impedances, modulator element frequency response and antenna gain is shown in Figure 6.

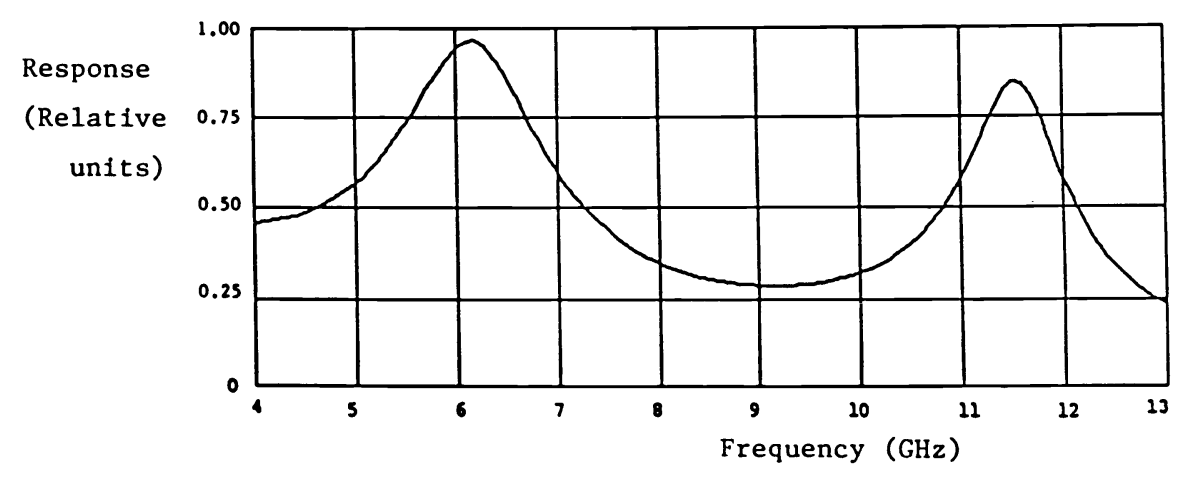

Figure 6. Frequency response predicted by simple model of antenna and electrode impedances, modulator element frequency response and antenna gain.

It appears that the peak around $12 \mathrm{GHz}$ occurs when the modulator elements are a half wave long, at which point the antennas are approximately two half-wavelengths long. The antenna drivingpoint impedance and the modulator element input impedance are then both high. At $6 \mathrm{GHz}$ there is another peak when the antenna is a half-wave long and the modulator element has a low input impedance. The lower-frequency peak also benefits from the increased modulator sensitivity at lower frequency - the modulator electrodes are electrically shorter and the sinc terms are nearer to unity. It was necessary to use WR 137 waveguide to measure this lower-frequency peak, so the microwave feed geometry was changed. Using the model above we find that there is a better fit to the data if we use 3.8 as the effective microwave refractive index instead of 4.3 .

Peak response values exceed 100 degrees per $\mathrm{W}^{1 / 2}$. This compares favorably with conventional microwave modulators (Erasme et al. ${ }^{7}$ report a peak response of 131 degrees per $\mathrm{W}^{1 / 2}$ for their phasereversal travelling-wave modulator, operating at a wavelength of $1153 \mathrm{~nm}$ - note that sensitivity varies as $\left.1 / \lambda_{\text {optical }}\right)$. This is only achieved over a narrow band, but is encouraging in a prototype. The 
narrow bandwidth results from the use of simple resonant elements. Other antenna designs with broadband elements will likely result in a flatter frequency response. Of course even here there is a region between the two peaks which is quite flat, but less sensitive by about $10 \mathrm{~dB}$.

The modulator was operated with a number of different wedge angles to establish the effect of the illumination angle on performance. This may be modeled by considering the pattern of each antenna alone, the pattern of a phased array of such antennas (with the light in the optical waveguide doing the phasing), and the pattern of a phased array of antennas with unequal illumination. Figure 7 shows the patterns of arrays of 3,4 and 5 equally illuminated antennas. We have included the smaller numbers

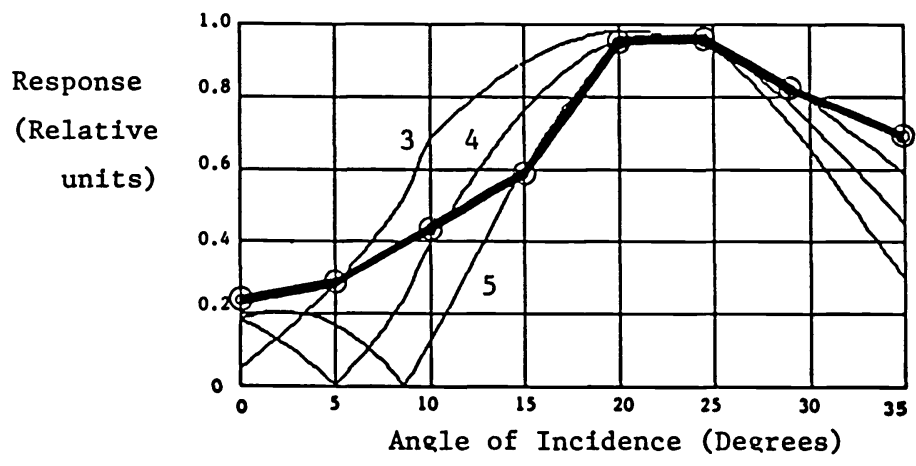

Figure 7. Effect of illumination angle on performance:

theory for 3,4, and 5 antennas, and results from experimental modulator. of antennas because the antennas at either end of the modulator may be poorly illuminated. If a cosine distribution across the five antennas is assumed, the result is not unlike the 4-antenna case. Also plotted in Figure 7 is the average response over the 9-12 GHz band of the prototype modulator at each angle of incidence. The response peaks at about $23^{\circ}$ as expected, confirming the phase-velocity-matching picture. The absence of the null in the experimental data is probably due to the multiple reflections inside the wedge.

A question related to the angle of incidence is the effect of having a non-planar modulating wave. In this case there is a phase error in the wave reaching each modulator. For example, if the wave is

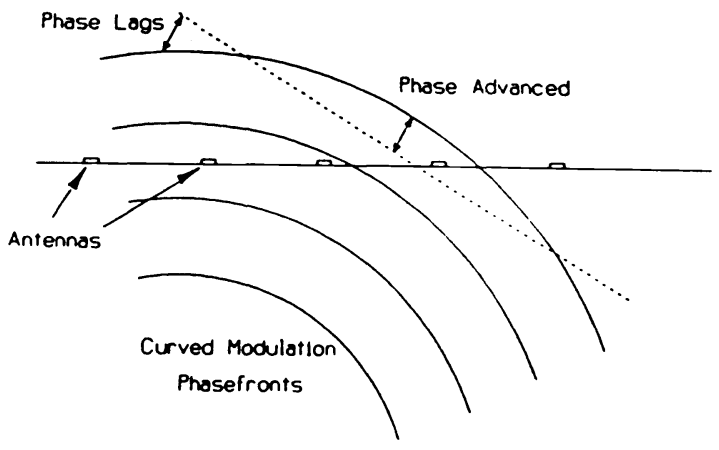

Figure 8. Effect of curved modulation phasefronts. spherical and diverging, the antennas toward the center of the modulator will be driven in a phase which is too far advanced, while those at the ends will be driven in a lagging phase (Figure 8). These effects result in some degradation of the response. For five antennas (spaced as they are in the prototype) a phase-front curvature of $30 \mathrm{~mm}$ results in $80 \%$ response, while a $90 \%$ response requires a 
curvature of about $45 \mathrm{~mm}$. We estimate that the phase-front curvature produced by the feed used is approximately $40 \mathrm{~mm}$. Clearly it will be important to maintain large radius phasefronts in future designs.

\section{FUTURE DEVELOPMENTS}

The theory of antennas on dielectric substrates is not yet well-developed, and improvements in this area should be applicable to this type of modulator. Improved waveguide-to-antenna coupling will also bring better performance. A $60 \mathrm{GHz}$ modulator mask with 30 electrode/antenna segments has been prepared and the modulator is currently being fabricated.

\section{ACKNOWLEDGEMENTS}

The authors wish to acknowledge the technical support of Robert L. Joyce and Reynold E. Johnson in this work, and many valuable discussions with Robert W. Terhune.

\section{REFERENCES}

[1] G. E. Betts, L. M. Johnson, and C. H. Cox, III, "High-Sensitivity Lumped-Element Bandpass Modulators in $\mathrm{LiNbO}_{3}$," IEEE J. Lightwave Tech., Vol. 7, pp 2078-2083, December 1989.

[2] C. M. Gee, G. D. Thurmond, and H. W. Yen, "17-GHz Bandwidth Electro-Optic Modulator," Appl. Phys. Lett., Vol. 43, pp 998-1000, December 1983.

[3] R. C. Alferness, S. K. Korotky, and E. A. J. Marcatili, "Velocity-Matching Techniques for Integrated Optic Traveling Wave Switch/Modulators," IEEE J. Quant. Electron., Vol. QE-20, pp 301309, March 1984.

[4] J. H. Schaffner, "Analysis of a Millimeter Wave Integrated Electro-optic Modulator with a Periodic Electrode," Paper 13 at SPIE OE-LASE Conference 1217, Proceedings, pp 101-110, Los Angeles, Calif., January 16-17, 1990.

[5] N. Enghetta, C. H. Papas, and C. Elachi, "Radiation Patterns of Interfacial Dipole Antennas," Radio Science, Vol. 17, pp 1557-1566, Nov.-Dec. 1982. 
[6] See, for example, Reference Data for ㅁadio Engineers, 5th Ed., Howard Sams, New York, 1968, Sec. 21-7.

[7] D. Erasme, D. A. Humphries, A. G. Roddie, and M. G. F. Wilson, "Design and Performance of Phase Reversal Traveling Wave Modulators," IEEE J. Lightwave Tech., Vol. 6, pp 933-936, June 1988. 